\title{
Imaging and Spectroscopy of Pristine and Cycled $\mathrm{Li}_{2} \mathrm{MnO}_{3}$
}

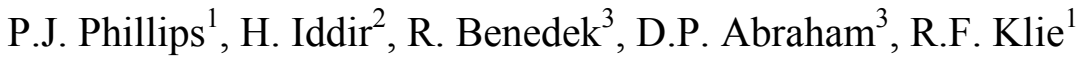 \\ ${ }^{1}$ Department of Physics, University of Illinois at Chicago, Chicago IL 60607. \\ ${ }^{2}$ Materials Science Division, Argonne National Laboratory, Argonne, IL 60439. \\ ${ }^{3}$ Chemical Sciences \& Engineering, Argonne National Laboratory, Argonne, IL 60439.
}

Li-ion batteries have yet to reach their full application potential due to their inherent cycling performance issues, such as voltage instability and capacity fading [1], while fundamental questions remain unanswered regarding the mechanisms responsible. However, Mn-based layered oxides continue to be promising candidates for cathodes in high-energy-density Li batteries given their high voltage and high discharge capacities [2]. A significant impediment to gaining insight into these layered oxides lies in their complexity and inhomogeneity. Thus, the focus of the present contribution will be on the parent material, $\mathrm{Li}_{2} \mathrm{MnO}_{3}$, which shows cycling characteristics similar to its more complex offspring. Scanning transmission electron microscopy (STEM) and spectroscopy are used to characterize structural and electronic properties of both pristine and cycled material.

An aberration-corrected JEOL JEM-ARM 200CF STEM instrument, capable of $0.73 \AA$ spatial and 0.35 $\mathrm{eV}$ energy resolution was used to examine $\mathrm{Li}_{2} \mathrm{MnO}_{3}$. STEM-based methods are quickly becoming the most promising characterization tools for these and similar materials, owed largely to the wide-range of techniques available on advanced STEM instruments, including the direct imaging of both heavy and light elements, and both energy-dispersive X-ray (EDX) and electron energy loss (EEL) spectroscopies. Imaging modes such as high/low angle annular dark field (H/LAADF) and annular bright field (ABF) are exploited to image heavy atomic columns, strain contrast, and light atomic columns, respectively. Additionally, electron energy loss spectroscopy along with calculations based on density functional theory are used to probe the local electronic structure by monitoring the $\mathrm{O} K$ - and $\mathrm{Mn} L$-edges, which can be used to track changes to both the $\mathrm{O}$ content and the Mn valence.

Some imaging and spectroscopy results acquired from pristine material are presented in Figs. 1 and 2. Fig. 1 contains an $\mathrm{Li}_{2} \mathrm{MnO}_{3} \mathrm{LAADF} / \mathrm{ABF}$ image pair taken along the [010] direction and an atomic model viewed along the same orientation. The [010] zone axis allows for the direct imaging of $\mathrm{O}$ columns, which are adjacent to the $\mathrm{Mn} / \mathrm{Li}$ mixed planes, and Li columns, which are sandwiched by the $\mathrm{O}$ columns; both $\mathrm{O}$ and $\mathrm{Li}$ are visible in the ABF image. An EEL spectrum acquired on pristine $\mathrm{Li}_{2} \mathrm{MnO}_{3}$ is presented in Fig. 2. As noted above, using key features such as the $\mathrm{O} K$-edge pre-peak intensity and the $\mathrm{Mn} L_{3 / 2}$ ratio and $L_{3}$ onset, we can track the electronic structure of $\mathrm{Li}_{2} \mathrm{MnO}_{3}$. Thus, the focus of this presentation will be on the structural and electronic evolution of the pristine layered oxide explored by combining spectroscopy and atomic-scale imaging with various in situ microscopy techniques and ex situ electrochemical cycling. Specifically, features such as atomic ordering of $\mathrm{Mn} / \mathrm{Li}$ atoms, $\mathrm{O}$ vacancy evolution, and $\mathrm{Mn}$ valence changes will be of particular interest.

\section{References}

[1] J. Zheng, M. Gu, P. Zuo, C. Wang, J.-G. Zhang, Nano Lett. 13 (2013) 3824-3830.

[2] M.M. Thackeray, C.S. Johnson, J.T. Vaughey, N. Li, S.A. Hackney, J. Mater. Chem. 15 (2005) 2257-2267. 
[3] The UIC JEOL JEM-ARM 200CF is supported by an MRI-R ${ }^{2}$ grant from the National Science Foundation (Grant No. DMR-0959470).
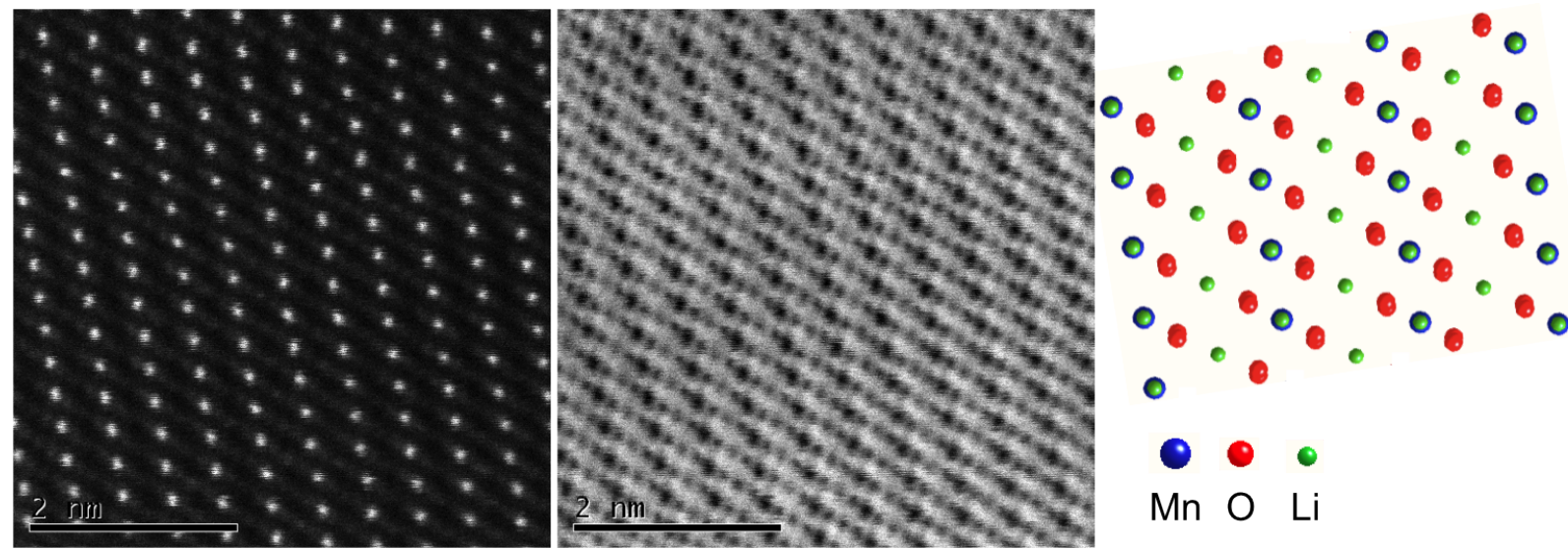

Figure 1: $\mathrm{LAADF} / \mathrm{ABF}$ image pair of pristine $\mathrm{Li}_{2} \mathrm{MnO}_{3}$ acquired along the [010] direction, along with a similarly-oriented atomic model. In the LAADF image, the mixed $\mathrm{Mn} / \mathrm{Li}$ columns appear as bright, with very faint intensity denoting $\mathrm{O}$ columns adjacent to these planes. We note the imaging of both $\mathrm{O}$ and $\mathrm{Li}$ columns in the ABF image.

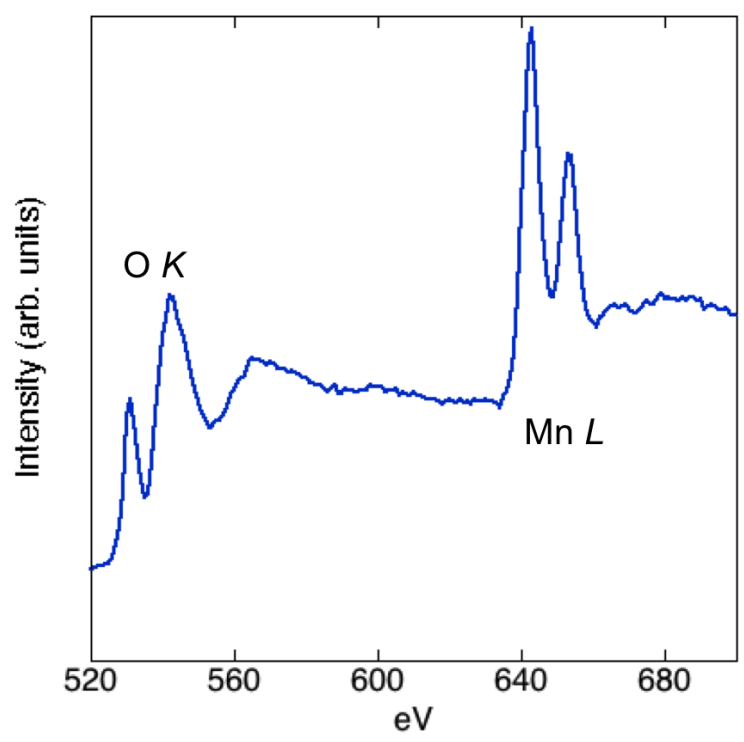

Figure 2: EELS results acquired on pristine $\mathrm{Li}_{2} \mathrm{MnO}_{3}$ of the $\mathrm{O} K$ - and $\mathrm{Mn} \mathrm{L}$ L-edges. Features relevant to classifying the electronic evolution of cycled material include the $\mathrm{O} K$-edge pre-peak intensity and the $\mathrm{Mn} L_{3 / 2}$ ratio and $L_{3}$ onset. 